\title{
ESTIMACIÓN DE LA PRODUCCIÓN SECUNDARIA DE PARAPRIONOSPIO PINNATA (SPIONIDAE, POLYCHAETA) FRENTE A BAHIA DE CONCEPCION, CHILE
}

\author{
FRANKLIN CARRASCO VAZQUEZ Y DAGOBERTO ARCOS ROJAS \\ Instituto de Biologia, Universidad de Concepción, Chile.
}

\section{SYNOPSIS}

The marine worm Paraprionospio pinnata population of a mud-bottom station at a depth of $65 \mathrm{~m}$ off Concepción Bay, Chile, has been studied over the period October 1976 to October 1977. The epscies were counted, divided into year groups and the biomass of each group es. timated as ash-free dry weight. Production estimate has been made by compunting mortality and residual biomass. Two year-class were present. The biomass, averaged over the year, was $1886 \mathrm{mg} / \mathrm{m}^{2}$ and the production $4529 \mathrm{mg} / \mathrm{m}^{2} /$ year, giving a P: B ratio of $2,4: 1$.

Introducción

Los estudios sobre la biota béntica marina, han recibido una buena atención en los últimos tiempos, en especial en el hemisferio norte. Los trabaios aue hacen referencia a los problemas de producción secundaria béntica no son abundantes. En el océano Pacífico sudoriental, el conocimiento es muy precario. Así en el marco de una investigación béntica, que se está realizando, frente a Bahía Concepción, Chile, se informa de algunos resultados preliminares. Este estudio está diseñado para evaluar la producción de un área, en la cual se desenvuelven importantes pesquerías regionales, como son por ejemplo las de los recursos demersales Merluccius gayi gayi y Pleuroncodes monodon.

Aspectos cuali y cuantitativos del ecosistema béntico en estudio, el cual comprende Bahía de Concepción, Golfo de Arauco y áreas adyacentes, han sido previamente tratados por Gallardo (1965-66) y Gallardo et al. (1972). El mismo autor (Gallardo, 1977) señala que la característica más notable de estas comunidades es la presencia de grandes comunidades microbianas, las cuales caracterizarían la biota de los fondos marinos que se encuentran bajo la influencia de las aguas ecuatoriales subsuperficiales. Por otro tado, Ahumada (1976), ha caracterizado las condiciones hidrográficas de Bahía de Concepción.

\section{Material y Metodos}

Paraprionospio pinnata (Ehlers, 1901), es un anélido poliqueto sedimentívoro (deposit-feeder), de amplia distribución, euribático, típico de fondos fangosos, fango-arcilla $y$ arcilla-arena (Foster, 1971). Este invertebrado es una de las formas dominantes en Bahía de Concepción (Gallardo et al., op. cit.) en la zona en estudio (ver Tabla I).

Para su estudio se realizó colectas preliminares, de donde se procedió a selecionar una estación, en la cual se aplicó la técnica de los muestreos repetidos. La estacióm elegida está situada $\left(73^{\circ} 06^{\prime} 35^{\prime \prime} \mathrm{W} ; 36^{\circ} 35^{\prime \prime} \mathrm{S}\right)$ frente a Bahía de Concepción, en la plataforma continental, con una profundidad de $65 \mathrm{~m}$. El sedimento es fango muy fino de color gris plomizo oscuro, mal oliente, debido a la presencia de $\mathrm{H}_{2} \mathrm{~S}$. Las muestras fueron colectadas entre octubre de 1976 y octubre de 1977 , realizándose ocho muestreos. Cada uno de ellos consistió en cuatro lances $\left(0,4 \mathrm{~m}^{2}\right)$ con una draga van Veen de $0,1 \mathrm{~m}^{2}$. La operación se realizó a bordo del B/C LUND de la Universidad de Concepción. La ubicación de la estación se efectuó por medio de radar, como también de demarcación visual. Las muestras fueron lavadas a bordo en un recipiente plástico de $60 \ell$, con agua de mar agitándose manualmente para poder así liberar el fino, pegajoso y compacto sedimento. El rebalse se recibió sobre tamices sedimentológicos de $0,250 \mathrm{~mm}$ de trama. Este sistema de lavado fue el que brindó mejores resultados, ya que no dañaba las pequeñas formas, no se incurría en pérdidas de organismos y se evitaba el conducir las muestras al laboratorio sin tamizar. A continuaçión se fijó las muestras con formalina al $10 \%$ y se tiffó con una solución acuosa de rosa de bengala. La siguiente etapa, en que se separó los ejemplares por taxa, fue la que demand6 mayor cantidad de tiempo, debido a que la microflora se presenta como largos filamentos, los cuales conforman una especie de conglomerado, del cual hay que desenredar los ejemplares, principalmente anélidos, labor muy delicada por el tamaño de algunos organismos. Así se separó todos los ejemplares y se identificó hasta el nivel de especie. Los especímenes de Paraprionospio pinnata. se dividió en clases anuales usando como mediò el ancho corporal máximo. La biomasa de cada grupo anual se estimó como peso seco sin cenizas, seguiéndose la metodología de Buchanan \& Warwick (1974) y Warwick \& Price (1975). Para la estimación de la producción de la especie se empleó el método de calculo que usa la mortalidad más la biomasa residual, señalado por Crisp (1971).

\section{Resultados}

$\mathrm{Al}$ analizar los histogramas de la frecuencia mensual de tallas (Fig. 1), se observa que existe en octubre de 1976 un gran grupo anual. En las muestras de diciembre de 1976 y enero de 1977 ya se vislumbran dos grupos modales, los que se consideró grupos anuales "O" y "1+". El grupo "1+" ya no está presente en marzo de 1977 mientras el grupo "O" continúa creciendo hasta completar el ciclo estudiado en octubre de 1977. Las proporciones de los grupos anuales presentes, específicamente en los casos de diciembre de 1976 y enero de 1977 , se extractó del análisis sobre papel de probabilidad compuesto (Harding, 1949; Cassie, 1954) y se presenta también en la Figura 1. Los pesos de las clases anuales se determinó derivando la relación entre el log de la amplitud ( $\mathrm{mm}$ ) del extremo anterior del cuerpo (ancho corporal máximo) y el log del peso seco sin cenizas (mg) de cada individuo. Para ello se eligió 40 especímenes intactos del mes de octubre de 1976, los que fueron medidos y pesados en forma individual. La ecuación de lá regresión es $\mathrm{Y}=0,258+4,152 \mathrm{X}$ $(r=+0,959)$. Los cálculos de la producción anual se presenta en la tabla a continuación, donde la producción secundária total alcanza a $4.529,46 \mathrm{mg} / \mathrm{m}^{2}$ /año.

Cálculos Producción Paraprionospio pinnata

\begin{tabular}{lrrlcrc} 
& \multicolumn{7}{c}{$\mathrm{N}\left(\mathrm{m}^{-2}\right)$} & $\mathrm{W}(\mathrm{mg})$ & $\Delta \overline{\mathrm{W}}(\mathrm{mg})$ & $\overline{\mathrm{N}}$ & $\overline{\mathrm{N}} \Delta \overline{\mathrm{W}}(\mathrm{mg})$ \\
OCT & 0 & 4.920 & 0,39700 & - & - & - \\
DIC & 1 & 2.094 & 0,22929 & - & - & - \\
ENE & 1 & 627 & 0,33652 & 0,10723 & 1.361 & 145,94 \\
DIC & 0 & 1.143 & 0,041350 & - & - & - \\
ENE & 0 & 5.423 & 0,07512 & 0,03377 & 3.282 & 110,86 \\
MAR & 0 & 23.200 & 0,05710 & - & - & - \\
MAY & 0 & 50.150 & 0,09532 & 0,03822 & 36.675 & $1.401,71$ \\
JUL & 0 & 26.670 & 0,14408 & 0,04876 & 38.410 & $1.872,87$ \\
AGO & 0 & 9.165 & 0,1517 & 0,00762 & 17.917 & 136,52 \\
OCT & 0 & 2.150 & 0,3040 & 0,1523 & 5.657 & 861,56
\end{tabular}

P $\quad 4.529 .46$

- Biomasa anual total $=15.090,4 \mathrm{mg}$

- Promedio anual-biomassa $=1.886 \mathrm{mg}$

$-\mathrm{P}: \mathrm{B}=2,4: 1$

Sobre la base de observaciones de la presencia o ausencia de gametos celómicos y por la dinámica de sus larvas en el plancton (Carrasco, 1976), P. pinnata debe empezar a liberar sus huevos, aproximadamente entre los meses de septiembre-octubre y persistir en ello hasta aproximadamente marzo. Este hecho va acompañado de una rapida mortalidad del grupo " $1+$ ", lo que se desprende del análisis de los histogramas de los meses de octubre de 1976 , diciembre del mismo afo y enero de 1977. En el mes de diciembre de 1976 apareœ claramente el grupo "O", mientras que el grupo " $1+$ " declina acentuadamente por mortalidad. El grupo "O" prosigue el reclutamiento prácticamente hasta mayo de 1977 al considerar la talla que presentan los organismos y esto se confirma al observar la Figura 2, ya que en dicho mes se encuentra el mayor número de individuos establecidos en el bentos y que corresponde al mayor valor de la biomasa total (ver Fig. 3). De la misma Figura 2 , se puede decir que después del máximo de mayo proviene una acentuada disminución del número de individuos, debido a mortalidad, por otra parte el crecimiento se incrementa gradualmente hasta alcanzar el máximo en octubre. 

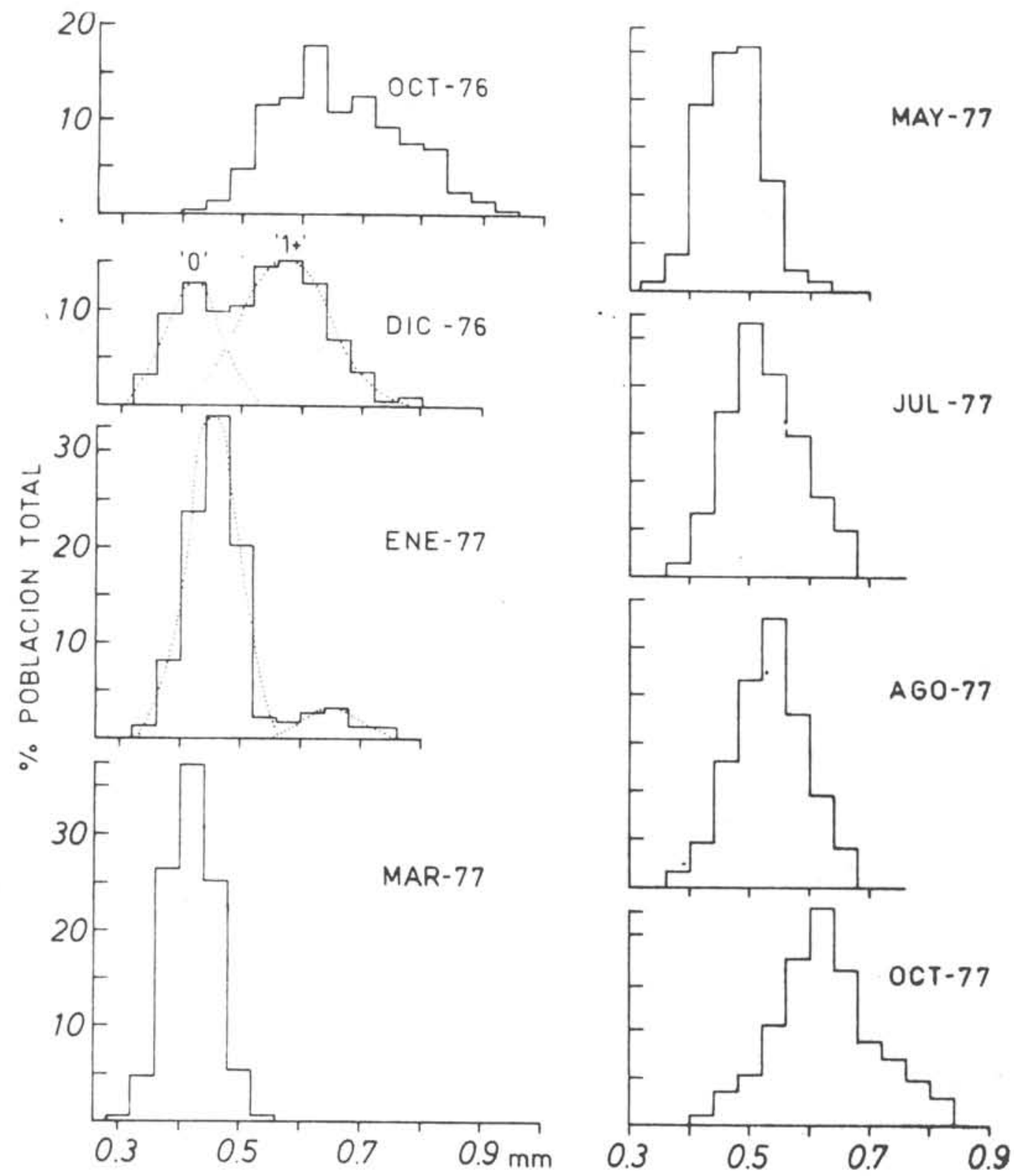

Fig. 1. Histogramas de la frecuencia de tallas de $P$. pinnataen los diferentes meses de muestreo.

TABLA I

\begin{tabular}{lrr} 
OCTUBRE $1976 \quad$ Número & $\%, 4 \mathrm{~m}^{2}$ & $\%$ \\
\hline Ampelisca araucana & 4508 & 33,11 \\
Mediomastus brauchiferus & 2153 & 15,81 \\
Paraprionospio pinnata & 1968 & 14,45 \\
Aricidea pigmentada & 1697 & 12,46 \\
Magelona phyllisae & 1051 & 7,72 \\
Cossura chilensis & 705 & 5,17 \\
Ancistrosyllis bassi & 603 & 4,42 \\
Nephtys ferruginea & 435 & 3,19 \\
Sosanides glandularis & 232 & 1,70 \\
Megalommu monoculata & 83 & 0,60 \\
Nereis dorsolobata & 51 & 0,37 \\
Anthozoa & 29 & 0,21
\end{tabular}

Phyllochaetopterus sp.

Harmothoe sp.

Lumbrineris sp.

Pectinaria chilensis

Pinnixa sp.

Pista sp.

Flabelligeridae

Glycera americana

Glycera longissima

Diopatra chiliensis

Mulinia sp

Pagurus villosus

Nemertino

Brachyura (megalopas)

\begin{tabular}{rl}
27 & 0,19 \\
20 & 0,14 \\
16 & 0,11 \\
11 & 0,08 \\
5 & 0,036 \\
4 & 0,029 \\
4 & 0,029 \\
3 & 0,022 \\
1 & 0,007 \\
1 & 0,007 \\
1 & 0,007 \\
1 & 0,007 \\
1 & 0,007 \\
2 & 0,014 \\
\hline 3619 & 99,805
\end{tabular}




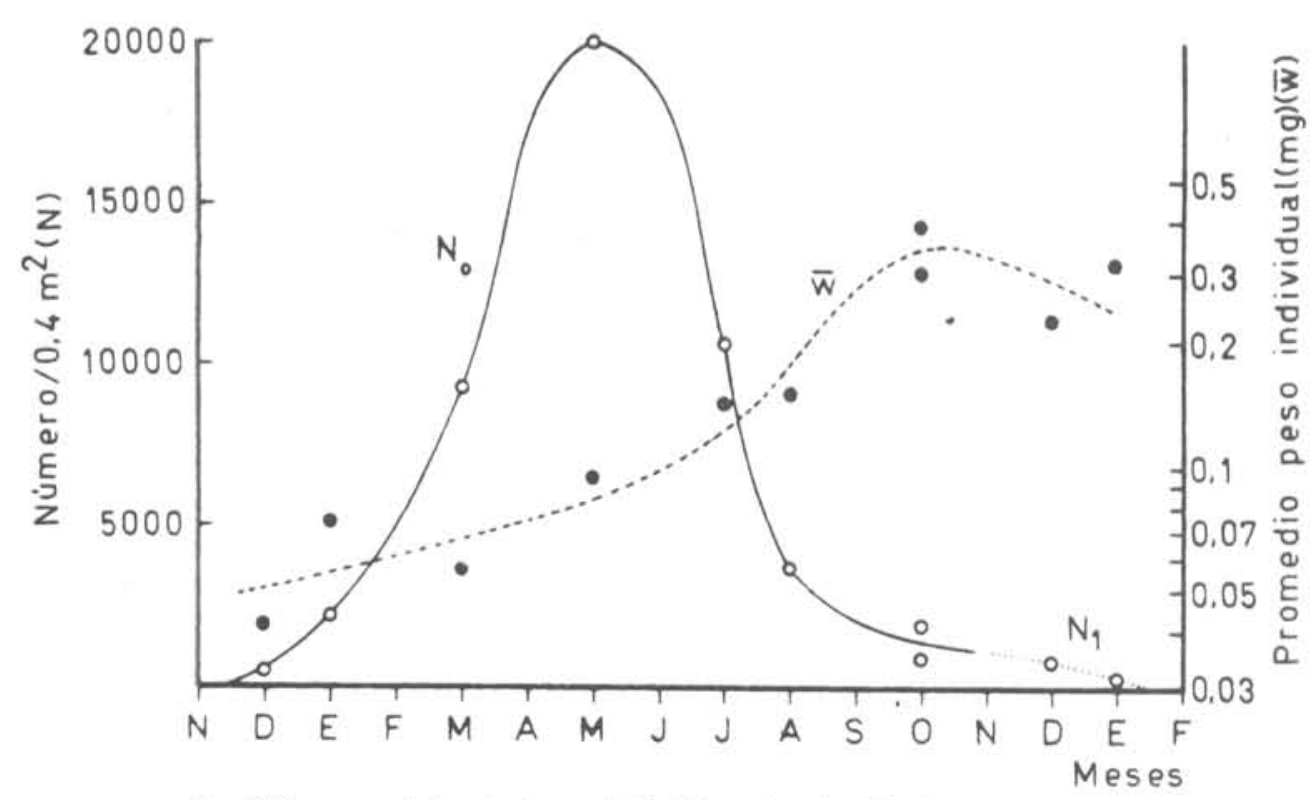

Fig. 2. Curvas reclutamiento-mortalidad (escala aritmética) y crecimiento (escala logarítmica). Octubre 1976 a octubre 1977.

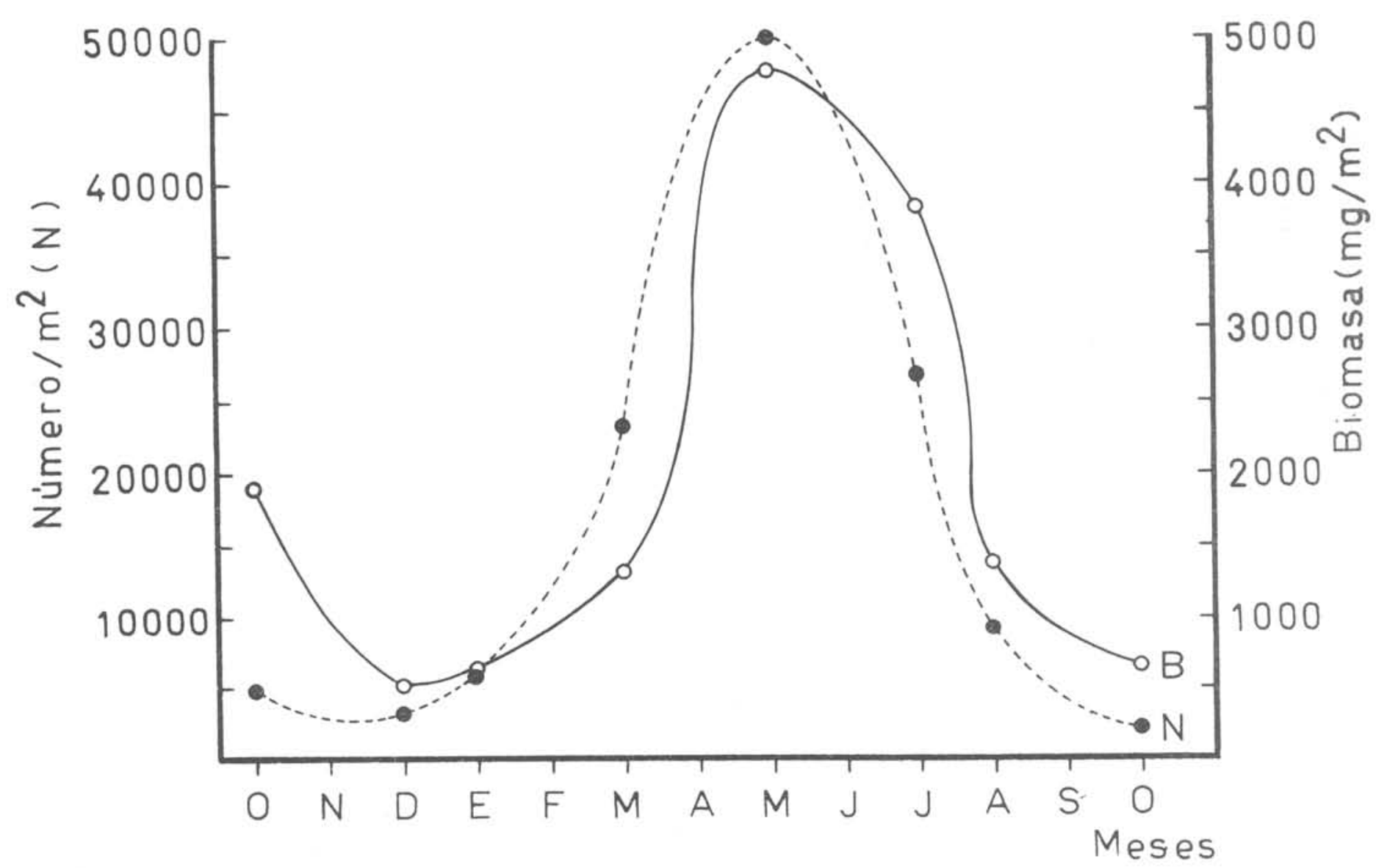




\section{Agradecimientos}

Vaya nuestro reconocimiento a la Asociación de Industriales Pesqueros de $\mathrm{T}$ alcahuano, quien en convenio con la Universidad de Concepción prestó su valiosa asistencia financiera, posibilitando la realización del presente estudio.

\section{Referencias Bibliográficas}

AHUMADA, R. B. 1976. Contribución al conocimiento de las condiciones hidrográficas de la Bahía de Concepción y áreas adyacentes (Chile). Tesis. Universidad de Concepción, 135p.

BUCHANAN, J. B. \& WARWICK, R. M. 1974. An estimate of benthic macrofaunal production in the offshore mud of the Northumberland coast. J. mar. biol. Ass. U. K., 54 (1): 197-222.

CARRASCO, F. D. 1976. Larvas de la familia Spionidae (Poly. chaeta) en el plancton de la Bahía de Concepción, Chile. Gayana, Zool., (38): 1-63.

CASSIE, R. M. 1954. Some uses of probability in the analysis of size frequency distributions. Aust. J. mar. Freshwat. Res., $5: 513-522$.
CRISP, D, J, 1971. Energy flow measurements, In: Holme, N. A. \& McIntyre, A. D., ed. - Methods for the study of marine benthos. Oxford, Blackwell, p. 197-279.

FOSTER, N. M. 1971. Spionidae (Polychaeta) of the Gulf of Mexico and the Caribbean Sea. Stud. Fauna Curaçao, 36 (129): $1-183$.

GALLARDO, V. A. 1965-66. Observaciones sobre la fauna bentónica del Golfo de Arauco. Boln Soc. Biol. Concepción, 40: $145-160$.

_._- 1977. Large benthic microbial communities in sulphide biota Peru-Chile Subsuface countercurrent. Nature, 268 : 331-332.

----; CASTILLO, J. G. \& YANEZZ, L. A. 1972. Algunas consideraciones preliminares sobre la ecología bentónica de los fondos sublitorales blandos en la Bahía de Concepción. Boln Soc. Biol. Concepción. 44:169-190.

HARDING, J. P. 1949. The use of probabilty paper for the graphi$\mathrm{cal}$ analysis of polymodal frequency distributions. J. mar. biol . Ass. U. K., 28: 141-153.

WARWICK, R. M. \& PRICE, R. 1975. Macrofauna production in an estuarine mud-flat. J. mar biol. Ass. U. K., 55 (1): $1-18$. 